\title{
EPIDEMIOLOGIA DO TRAUMATISMO DA COLUNA VERTEBRAL
}

\section{EPIDEMIOLOGY OF SPINE INJURIES}

\author{
Marcelo Ferraz de Campos ${ }^{1}$; André Tosta Ribeiro; \\ Sérgio Listik; ; Clemente Augusto de Brito Pereira ${ }^{4}$; \\ Jozias de Andrade Sobrinho, ECBC-SP ${ }^{5}$; Abrão Rapoport, ECBC-SP ${ }^{5}$
}

\begin{abstract}
RESUMO: Objetivo: Avaliação epidemiológica retrospectiva de 100 casos de traumatismo da coluna vertebral. Método: Estudo transversal de dados colhidos por levantamento de prontuário, segundo protocolo de decodificação local. Resultados: Predomínio etário de 20 a 40 anos em 64\% dos casos; sexo masculino em $86 \%$; segmento toracolombar mais comumente atingido $64 \%$ e $36 \%$ para o segmento cervical; principais causas foram às quedas em $40 \%$, seguidas de acidentes automobilísticos em $25 \%$ e quedas da laje $23 \%$. A prevalência dos ferimentos por arma de fogo foi de $7 \%$, mergulho em águas rasas $3 \%$ e agressões $2 \%$. Houve análise complementar com cruzamentos entre idade, sexo, causa e segmento da coluna vertebral acometido, observando que o segmento cervical teve grande predomínio nas mulheres em relação aos homens em 85,7\% X 14,3\%. Conclusão: O traumatismo da coluna vertebral ocorreu predominantemente em homens entre 20 e 40 anos e o segmento cervical foi o mais acometido nas mulheres em relação aos homens na proporção de 6:1 (Rev. Col. Bras. Cir. 2008; 35(2): 088-093).
\end{abstract}

Descritores: Fraturas da Coluna Vertebral/epidemiologia; Traumatismos da Medula Espinal; Acidente; Coluna vertebral.

\section{INTRODUÇÃO}

O traumatismo da coluna vertebral com lesão irreversível da medula é agudo e inesperado que altera drasticamente a vida do indivíduo acometido, gerando desastrosas consequiências ao lesado, à família e à sociedade. A importância de trabalhos epidemiológicos sobre pacientes com lesão medular aguda torna-se evidente quando observamos que a única forma de melhorar a qualidade de vida da população, e diminuir os custos com estes pacientes, é evitar que as complicações aconteçam.

O trauma raquimedular compreende as lesões dos componentes da coluna vertebral em quaisquer porções: óssea, ligamentar, medular, discal, vascular ou radicular. Nos Estados Unidos, aproximadamente $15 \%$ dos pacientes com trauma de coluna vertebral terão comprometimento neurológico, persistindo como melhor conduta a prevenção ${ }^{1}$.

A incidência de lesão medular traumática no Brasil é desconhecida, pois esta condição não é sujeita à notificação e há poucos dados e trabalhos publicados a respeito da epidemiologia da lesão medular. Estima-se que ocorram a cada ano no país, mais de 10.000 novos casos de lesão medular, sendo o trauma a causa predominante ${ }^{2}$. Assim, realizamos um estudo demográfico que poderá proporcionar um conhecimento mais preciso do grupo de risco e das causas do traumatismo raquimedular em nossa população e compará-las com as da literatura, visando principalmente estabelecer um grupo de risco e sugerir programas de prevenção adequados a essa afecção.

Objetivamos estudar a freqüência dos traumatismos raquimedulares na população atendida num hospital público da região Sul de São Paulo, segundo a idade, sexo, causa e segmento da coluna acometido.

\section{MÉTODO}

Estudo transversal baseado na análise de 100 pacientes com traumatismo da coluna vertebral, diagnosticados e tratados, sendo que os dados foram obtidos do protocolo de Traumatismo da Coluna Vertebral.

Levou-se em conta as seguintes variáveis: sexo; idade (separadas pelas faixas etárias de 0 a 20, 21 a 30, 31 a 40, 41 a 50, 51 a 80); causas do acidente: quedas gerais, quedas de andaimes, da escada, da própria altura, da árvore, do telhado, da ponte, do viaduto), acidentes com veículos, ferimentos por arma de fogo, mergulhos em água rasa e agressões; segmento (cervical ou toracolombar).

Os dados foram analisados com o auxílio do programa digital "Windows Excel" para a obtenção das freqüências absoluta e relativa das variáveis e a associação destas pelo teste qui-quadrado, com nível de significância de 5\%, quando cruzadas as variáveis idade-sexo, causa-sexo, segmento-sexo,

1. Neurocirurgião do Departamento de Neurocirurgia do Hospital Heliópolis, Hosphel, São Paulo.

2. Residente do Departamento de Neurocirurgia do Hospital Heliópolis, Hosphel, São Paulo.

3. Neurocirurgião do Departamento de Neurocirurgia Estereotáxica e Funcional do Hospital Brigadeiro, São Paulo.

4. Chefe do Departamento de Neurocirurgia do Hospital Heliópolis, Hosphel, São Paulo.

5. Professor do Curso de Pós Graduação em Ciências da Saúde do Hospital Heliópolis, Hosphel, São Paulo.

Recebido em 22/10/2007

Aceito para publicação em 02/12/2007

Conflito de interesses: nenhum

Fonte de financiamento: nenhuma

Trabalho realizado no do Departamento de Neurocirurgia do Hospital Heliópolis, Hosphel, São Paulo. 
causa-idade, segmento-idade e causa-segmento. Este teste não foi utilizado quando havia muitas caselas com frequiência de zero.

\section{RESULTADOS}

A principal causa foi a queda geral com $40 \%$, seguida por acidentes automobilísticos $(25 \%)$, quedas de laje $(23 \%)$, ferimentos por arma de fogo $(7 \%)$, mergulhos em águas rasas (3\%) e agressões $(2 \%)$.

Ao cruzar sexo com idade (Tabela 1), observou-se que a maioria dos acidentes ocorreu em indivíduos do sexo masculino (86\%), com predomínio em jovens, e para ambos os gêneros pouco mais de $40 \%$ dos acidentes ocorreram em pessoas com menos de 30 anos.

Ao se estudar as causas (Tabela 2), o sexo masculino contou com $43,0 \%$ das quedas gerais, enquanto que o sexo feminino representou $21,4 \%$. Verificou-se que a principal causa de acidente no sexo feminino foram os acidentes automobilísticos $(50,0 \%)$. No sexo feminino, não houve caso de ferimento por arma de fogo, mergulho em águas rasas e agressões, enquanto que, no sexo masculino, estes acidentes ocorreram em 12,7\% dos casos. Ainda (Tabela 3), houve acometimento do segmento cervical em $39,5 \%$ dos casos no sexo masculino contra $14,3 \%$ no sexo feminino. Para o segmento toracolombar, houve $60,5 \%$ de casos no sexo masculino e $85,7 \%$ no sexo feminino. Para todas as avaliações representadas nas Tabelas 1, 2 e 3 não houve diferença estatística entre os sexos.

Quando se analisa o cruzamento de idade e a causa (Tabela 4), as principais causas de acidentes em indivíduos de 0 a 20 anos foram quedas de laje $(35,0 \%)$ e acidentes automobilísticos $(25,0 \%)$. Na faixa etária de 21 a 30 anos, o predomínio foi de acidentes automobilísticos $(50,0 \%)$ e quedas $(29,2 \%)$. Em indivíduos de 31 a 40 anos, as principais causas foram quedas (40\%), acidentes automobilísticos $(30 \%)$ e quedas de lajes (25\%). Na faixa de 41 a 50 anos, os acidentes foram quedas de lajes $(44,4 \%)$ e quedas gerais

Tabela 1 - Número e porcentagem de pacientes segundo sexo e idade.

\begin{tabular}{|c|c|c|c|c|c|c|}
\hline \multirow{2}{*}{$\begin{array}{c}\text { Idade, Causa e Segmento } \\
\text { Idade } \\
0\end{array}$} & \multicolumn{4}{|c|}{ Sexo } & \multirow{2}{*}{\multicolumn{2}{|c|}{$\frac{\operatorname{Total}^{\mathbf{0}}(\%)}{\text { Total }^{\circ}(\%)}$}} \\
\hline & \multicolumn{2}{|c|}{ Masculino $\mathbf{N}^{\circ}(\%)$} & \multicolumn{2}{|c|}{ Feminino $N^{\circ}(\%)$} & & \\
\hline $0-20$ & 17 & $(19,8)$ & 3 & $(21,4)$ & 20 & $(20,0)$ \\
\hline $21-30$ & 20 & $(23,3)$ & 4 & $(28,6)$ & 24 & $(24,0)$ \\
\hline $31-40$ & 19 & $(22,1)$ & 1 & $(7,1)$ & 20 & $(20,0)$ \\
\hline $41-50$ & 15 & $(17,4)$ & 3 & $(21,4)$ & 18 & $(18,0)$ \\
\hline $51-80$ & 15 & $(17,4)$ & 3 & $(21,4)$ & 18 & $(18,0)$ \\
\hline Total & 86 & $(100,0)$ & 14 & $(100,0)$ & 100 & $(100,0)$ \\
\hline
\end{tabular}

Valor de $p=0,788$.

Tabela 2 - Número e porcentagem de pacientes segundo sexo e causa.

\begin{tabular}{|c|c|c|c|c|c|c|}
\hline \multicolumn{7}{|c|}{ Sexo } \\
\hline \multirow{2}{*}{$\begin{array}{l}\text { Causa } \\
\text { Quedas gerais }\end{array}$} & \multicolumn{2}{|c|}{ Masculino $\mathrm{N}^{\circ}(\%)$} & \multicolumn{2}{|c|}{ Feminino $N^{\circ}(\%)$} & \multicolumn{2}{|c|}{ Total $N^{\circ}(\%)$} \\
\hline & 37 & $(43,0)$ & 3 & $(21,4)$ & 40 & $(40,0)$ \\
\hline Acidente automobilístico & 18 & $(20,9)$ & 7 & $(50,0)$ & 25 & $(25,0)$ \\
\hline Quedas de lajes & 19 & $(22,1)$ & 4 & $(28,6)$ & 23 & $(23,0)$ \\
\hline Ferimento por arma de fogo & 7 & $(8,1)$ & - & $(-)$ & 7 & $(7,0)$ \\
\hline Mergulho em águas rasas & 3 & $(2,3)$ & - & $(-)$ & 3 & $(3,0)$ \\
\hline Agressões & 2 & $(2,3)$ & - & $(-)$ & 2 & $(2,0)$ \\
\hline Total & 86 & $(100,0)$ & 14 & $(100,0)$ & 100 & $(100,0)$ \\
\hline
\end{tabular}

Sem teste estatístico por haver caselas em branco.

Tabela 3 - Número e porcentagem de pacientes segundo sexo e segmento.

\begin{tabular}{|c|c|c|c|}
\hline \multicolumn{4}{|c|}{ Sexo } \\
\hline Segmento & Masculino $\mathbf{N}^{\circ}(\%)$ & Feminino $\mathrm{N}^{\circ}(\%)$ & Total $N^{\circ}(\%)$ \\
\hline Cervical & $34 \quad(39,5)$ & $2 \quad(14,3)$ & $36 \quad(36,0)$ \\
\hline Toracolombar & $52 \quad(60,5)$ & $12 \quad(85,7)$ & $64 \quad(64,0)$ \\
\hline Total & $86 \quad(100,0)$ & $14 \quad(100,0)$ & $100(100,0)$ \\
\hline
\end{tabular}

$p=0,068$. 
(38\%). Por fim, na faixa etária de 51 a 80 anos os acidentes foram, em sua grande maioria, as quedas $(83,3 \%)$.

Ao avaliar o segmento acometido em relação à idade (Tabela 5), o segmento cervical foi acometido em $45 \%$ da faixa etária de 0 a 20 anos, 37,5\% na faixa de 21 a 30 anos, $50 \%$ na faixa etária de 41 a 50 anos, 16,7\% para os de 41 a 50 anos e $27,8 \%$ no segmento de 51 a 80 anos. O segmento da coluna toracolombar foi acometido em $55 \%$ na faixa etária de 0 a 20 anos, $62,5 \%$ na faixa entre 21 a 30 anos, $50 \%$ nos de 31 a 40 anos, $83,3 \%$ na faixa etária de 41 a 50 anos e 72,2\% para aqueles de 51 a 80 anos. Para todas as avaliações representadas na tabela 2 não houve diferença estatística entre as faixas etárias.

Nos acidentes com acometimento no segmento cervical (tabela 6), houve predomínio de quedas $(30,6 \%)$ e quedas da laje $(27,8 \%)$. Já nos acidentes com acometimento no segmento toracolombar $45,3 \%$ foram quedas gerais e $28,1 \%$ foram acidentes automobilísticos.

\section{DISCUSSÃO}

São poucos os eventos no campo da Neurocirurgia que requerem do especialista tanto empenho e atenção quanto o trauma raquimedular. Por ser de instalação súbita e inesperada, torna-se um infortúnio de proporções desastrosas ao doente e à sua família, além de promover altos custos hospitalares à sociedade 3 . Nos Estados Unidos, por exemplo, há cerca de 150.000 pessoas vivendo incapacitadas tendo sofrido este tipo de trauma com acréscimo de aproximadamente 10.000 novos inválidos anualmente ${ }^{4}$. As internações prolongadas ainda contribuem para complicações clínicas ${ }^{5}$, acrescendo morbidez como: infecção respiratória, úlceras de decúbito, trombose venosa e embolia pulmonar.

Os crescentes avanços no tratamento dessas lesões, devido ao desenvolvimento e treinamento de equipes especializadas no atendimento aos pacientes politrauma-

Tabela 4 - Número e porcentagem de pacientes segundo idade e causa.

\begin{tabular}{|c|c|c|c|c|c|c|c|c|c|c|}
\hline \multirow{3}{*}{$\begin{array}{l}\text { Causa e Segmento } \\
\text { Causa } \\
\text { Quedas gerais }\end{array}$} & \multicolumn{8}{|c|}{ Idade } & \multicolumn{2}{|c|}{ Total } \\
\hline & \multicolumn{2}{|r|}{ 0-20 } & 21-30 & \multicolumn{2}{|c|}{$31-40$} & \multicolumn{2}{|c|}{ 41-50 } & $51-80$ & \multicolumn{2}{|c|}{ Total } \\
\hline & 3 & $(15,0)$ & $7 \quad(29,2)$ & 8 & $(40,0)$ & 7 & $(38,9)$ & $15 \quad(83,3)$ & 40 & $(40,0)$ \\
\hline Acidente automobilístico & 5 & $(25,0)$ & $12(50,0)$ & 6 & $(30,0)$ & 1 & $(5,6)$ & $(5,6)$ & 25 & $(25,0)$ \\
\hline Quedas de lajes & 7 & $(35,0)$ & $1 \quad(4,2)$ & 5 & $(25,0)$ & 8 & $(44,4)$ & $2(11,1)$ & 23 & $(23,0)$ \\
\hline Ferimento por arma de fogo & 4 & $(20,0)$ & $3(12,5)$ & - & $(-)$ & - & $(-)$ & $-\quad(-)$ & 7 & $(7,0)$ \\
\hline Mergulho em águas rasas & 1 & $(5,0)$ & $-\quad(-)$ & 1 & $(5,0)$ & 1 & $(5,6)$ & $-\quad(-)$ & 3 & $(3,0)$ \\
\hline Agressões & - & $(-)$ & $1 \quad(4,2)$ & - & $(-)$ & 1 & $(5,6)$ & $-\quad(-)$ & 2 & $(2,0)$ \\
\hline Total & 20 & $(100,0)$ & $24(100,0)$ & 20 & $(100,0)$ & 18 & $(100,0)$ & $18(100,0)$ & 100 & $(100,0)$ \\
\hline
\end{tabular}

Sem teste estatístico para idade e causa, por haver caselas em branco.

Tabela 5 - Número e porcentagem de pacientes segundo idade e segmento.

\begin{tabular}{|c|c|c|c|c|c|c|c|c|c|}
\hline \multirow{3}{*}{$\frac{\text { Segmento }}{\text { Cervical }}$} & \multicolumn{8}{|c|}{ Idade } & \multirow[b]{2}{*}{ Total } \\
\hline & & $0-20$ & 21-30 & & $31-40$ & & 41-50 & $51-80$ & \\
\hline & 9 & $(45,0)$ & $9 \quad(37,5)$ & 10 & $(50,0)$ & 3 & $(16,7)$ & $5 \quad(27,8)$ & $36 \quad(36,0)$ \\
\hline Toracolombar & 11 & $(55,0)$ & $15 \quad(62,5)$ & 10 & $(50,0)$ & 15 & $(83,3)$ & $13 \quad(72,2)$ & $64 \quad(64,0)$ \\
\hline Total & & $(100,0)$ & $24(100,0)$ & 20 & $(100,0)$ & 18 & $(100,0)$ & $18(100,0)$ & $100(100,0)$ \\
\hline
\end{tabular}

$p=0,209$.

Tabela 6 - Número e porcentagem de pacientes segundo causa e segmento.

\begin{tabular}{|c|c|c|c|c|c|c|}
\hline \multirow{3}{*}{$\begin{array}{l}\text { Causa } \\
\text { Causa } \\
\text { Quedas gerais }\end{array}$} & \multicolumn{4}{|c|}{ Segmento } & \multirow{2}{*}{\multicolumn{2}{|c|}{$\begin{array}{c}\text { Total } \\
\text { Total } \\
\end{array}$}} \\
\hline & \multicolumn{2}{|c|}{ Cervical } & \multicolumn{2}{|c|}{ Toracolombar } & & \\
\hline & & $(30,6)$ & 29 & $(45,3)$ & 40 & $(40,0)$ \\
\hline Acidente automobilístico & 7 & $(19,4)$ & 18 & $(28,1)$ & 25 & $(25,0)$ \\
\hline Quedas de lajes & 10 & $(27,8)$ & 13 & $(20,3)$ & 23 & $(23,0)$ \\
\hline Ferimento por arma de fogo & 4 & $(11,1)$ & 3 & $(4,7)$ & 7 & $(7,0)$ \\
\hline Mergulho em águas rasas & 3 & $(8,3)$ & - & $(-)$ & 3 & $(3,0)$ \\
\hline Agressões & 1 & $(2,8)$ & 1 & $(1,6)$ & 2 & $(2,0)$ \\
\hline Total & & $(100,0)$ & 64 & $(100,0)$ & 100 & $(100,0)$ \\
\hline
\end{tabular}

Nota: não foi feito o teste estatístico, pois há caselas em branco. 
tizados nos principais centros urbanos, proporcionam maior expectativa de sobrevida, inclusive nos casos mais graves, além da redução de complicações. No entanto, o prognóstico depende de uma longa fase de reabilitação para a reintegração do indivíduo à sociedade. A melhor conduta, portanto, passa a ser a prevenção, daí o grande valor aos estudos epidemiológicos.

Dados nacionais são escassos, estimando-se que o índice médio esteja por volta de 71 novos casos por milhão de habitantes ${ }^{2,6}$. Os números superam a maioria das estatísticas publicadas referentes à incidência da lesão medular em outros países por volta de 40 novos casos por um milhão de habitantes/ ano $^{7}$. Portanto, infere-se que, tais lesões tenham origem traumática, sendo ferimentos por armas de fogo (FAF), acidentes automobilísticos e quedas, as causas externas mais freqüentes ${ }^{8}$. As vítimas são predominantemente adultos jovens, entre 18 e 35 anos e na proporção de quatro homens para uma mulher ${ }^{8}$.

Os indivíduos mais propensos ao traumatismo da coluna vertebral dos tratados, são os homens, com $86 \%$ da casuística. Vários trabalhos que avaliam o sexo concordam com o resultado de nosso estudo ${ }^{9-23}$. No entanto, os traumas raquimedulares vêm aumentando sua incidência nas mulheres com o passar dos anos ${ }^{24}$. Na nossa casuística encontramos, entre as mulheres, grande predomínio de trauma no segmento cervical $(85,7 \%)$.

Em estudo realizado na região norte de $\mathrm{São} \mathrm{Paulo}^{25}$, a idade média encontrada foi de 35 anos, mais próxima da média encontrada ${ }^{17,23}$. Encontramos predominância da faixa de 21 a 30 anos (para o sexo masculino), condizente com outros dados da literatura ${ }^{15,18,19,22}$. No sexo feminino observamos que a maioria dos casos ocorreu abaixo dos $30 \operatorname{anos}^{12}$.

Em nosso Serviço, as causas principais de traumatismo da coluna vertebral foram predominantemente ocasionadas pelas quedas com $40 \%$, seguidas dos acidentes automobilísticos (25\%) e das quedas da laje (23\%.) Em menor freqüência, encontramos os ferimentos por arma de fogo, em torno de $7 \%$, os mergulhos em águas rasas (3\%) e as agressões $(2 \%)$.

Em um estudo do Hospital das Clínicas de São Paulo da década de $90^{11}$ sobre traumatismo da coluna vertebral, as principais causas foram ferimentos por arma de fogo $(36,7 \%)$, acidentes de trânsito $(26,9 \%)$, quedas gerais $(22,4 \%)$, mergulho $(7,7 \%)$ e outras $(6,5 \%)$. Observa-se que este trabalho não chama atenção para as quedas da laje como uma das causas do traumatismo na coluna vertebral, além de se aproximar mais das casuísticas norte-americanas, evidenciado por ordem de freqüência: os acidentes automobilísticos (45\%), as quedas $(22 \%)$, os esportes $(14 \%)$ e as lesões associadas à violência $(14 \%)^{26}$. Outros dados discrepantes estão presentes ${ }^{27}$ na literatura que refere o predomínio na idade de 15 a 19 anos e maior incidência para mergulho em águas rasas(45\%).

$\mathrm{Na}$ avaliação da causa em relação ao sexo, as quedas tiveram relativa prevalência no sexo masculino em 43,0\%. Para o sexo feminino, grande importância é dada à prevalência de acidentes automobilísticos $(50,0 \%)$. Quanto às quedas da laje, o índice é muito semelhante entre os sexos com $22,1 \%$ no masculino e $28,6 \%$ no feminino, sendo importante ressaltar que em nossa revisão não encontramos estudos cruzando essas duas variáveis. Tais dados são muito relevantes uma vez que auxiliariam no direcionamento dos principais objetivos de uma campanha preventiva.

Quando se considera o segmento vertebral acometido, $36 \%$ dos nossos pacientes apresentaram lesão cervical enquanto que $64 \%$ foram lesões toracolombares. Em contrapartida, os dados da literatura evidenciam maior prevalência de lesão cervical ${ }^{14,20,24,27}$, sendo os que mais se aproximam $^{26,28}$ da nossa casuística relatam $50 \%$ das lesões localizadas na região cervical.

Entretanto, ao analisarmos as causas em relação ao segmento acometido, constata-se que $65 \%$ das causas são decorrentes de quedas gerais e acidentes automobilísticos, reconhecidas causas de lesão toracolombar. Um dado curioso $^{2}$ é a ingesta alcoólica presente em $27,9 \%$ dos traumas com fratura toracolombar. Nas vítimas de trauma raquimedular cervical, a queda de laje foi a causa mais freqüente neste segmento da coluna ${ }^{3}$.

A estratificação das causas de acordo com faixas etárias predeterminadas permite melhor compreensão a respeito do segmento vertebral acometido, já que essas variáveis estão fortemente interligadas quando se considera o mecanismo de trauma e as atividades habituais a cada faixa etária.

Em nossa casuística, por exemplo, ao considerarmos indivíduos de 0 a 20 anos, quedas da laje perfizeram 35\% dos acidentes (região da cidade com predomínio de população carente que vive em terrenos ocupados de forma irregular), sendo o segmento cervical acometido em $45 \%$ do grupo. Esses parâmetros adquirem nova importância quando analisamos indivíduos de 21 a 30 anos, em que há predomínio de acidentes automobilísticos $(50 \%)$ e quedas $(29,2 \%)$, refletindo numa maior porcentagem de lesões toracolombares $(62,5 \%)$. A literatura refere trabalhos que fizeram essa comparação $0^{2,8,13,23,29,30}$, com indivíduos de 20 a 40 anos que foram as maiores vítimas de traumatismo toracolombar, tendo como principais causas a queda de altura com $44,06 \%$ e o acidente automobilístico com $28,81 \%$. Eles concluíram que, a grande maioria dos traumatismos da coluna torácica e lombar pode ser evitada com a observação dos itens de segurança do trabalho, no trânsito e no lar.

Ao longo deste estudo, procuramos apontar uma condição relativamente comum entre os pacientes com traumatismo da coluna vertebral. Evidenciamos que lesões graves como as cervicais, em que há maior risco de invalidez, ocorrem em indivíduos jovens que representam força de trabalho significante da sociedade, elevando, portanto, os gastos públicos no suporte a tais vítimas ${ }^{31-33}$.

Torna-se imperativa a programação de campanhas de prevenção a quedas e demais acidentes correlatos para essa situação em estudo. Cabe ainda às autoridades legais a fiscalização em maior empenho das regras de trânsito e da construção civil, a fim de se coibir o projeto de áreas residenciais elevadas sem ao menos alguma proteção às quedas, principalmente aquelas de laje, comuns em residências inacabadas em conjuntos habitacionais de populações carentes, onde a criança circula livremente e sem controle dos pais. Este tipo de acidente merece atenção especial das autoridades, considerados os traumas graves que acometem a população de baixa renda. 


\begin{abstract}
Background: Retrospective epidemiological analysis of 100 patients with trauma spine injury. Method: Data were collected through a local spine injury protocol, performing a transversal model. Results: Major incidence varied from 20 to 40 years old (64\% cases); $86 \%$ were male; thoracolumbar segment was the most injured segment (64\%) whereas cervical segment counted for $36 \%$. Simple falls were the most important cause, followed by car crash accident (25\%) and complex falls (23\%). Fire arm injuries counted for $7 \%$, shallow dives for $3 \%$ and aggressions for $2 \%$. Additional analysis between age, sex, cause, and spine segment was made. We found a greater incidence of cervical injury in females when in comparison to males $(85.7 \%$ versus 14.3\%). Conclusion: Spine injuries, prevailed in males between 20 and 40 years old, and the cervical segment was prevalent in women in a $6: 1$ proportion.
\end{abstract}

Key words: Spinal fractures/epidemiology; Spinal cord injuries; Accident; Spine.

\section{REFERÊNCIAS}

1. Boer VHT. Trauma raquimedular. In: Kruel NF, Araújo PA, editores. Manual de terapêutica-cirurgia. Florianópolis: Associação Catarinense de Medicina; 1997; p. 166-71.

2. Masini M. Tratamento das fraturas e luxações da coluna toracolombar por descompressão póstero-lateral e fixação posterior com retângulo e fios segmentares sublaminares associados a enxerto ósseo [dissertação]. São Paulo (SP): Escola Paulista de Medicina; 2000.

3. Sekhon LH, Fehlings MG. Epidemiology, demographics, and pathophysiology of acute spinal cord injury. Spine. 2001; 26(24 Suppl):S2-12.

4. Pinto LG. Lesões traumáticas raquimedulares agudas: conceitos atuais. Rev Col Bras Cir. 1982; 9(3):116-9.

5. da Paz AC, Beraldo PS, Almeida MC, Neves EG, Alves CM, Khan P. Traumatic injury to the spinal cord. Prevalence in Brazilian hospitals. Paraplegia. 1992; 30(9): 636-40.

6. Masini M. Estimativa da incidência e prevalência de lesão medular no Brasil. J Bras Neurocirurg. 2001; 12(2):97-100.

7. Blumer CE, Quine S. Prevalence of spinal cord injury: an international comparison. Neuroepidemiology. 1995; 14(5):25868.

8. Waters RL, Adkins RH. Firearm versus motor vehicle related spinal cord injury: preinjury factors, injury characteristics, and initial outcome comparisons among ethnically diverse groups. Arch Phys Med Rehabil. 1997; 78(2):150-5.

9. Aito S; Gruppo Italiano Studio Epidemiologico Mielolesioni GISEM Group. Complications during the acute phase of traumatic spinal cord lesions. Spinal Cord. 2003; 41(11):629-35.

10. Avanzi O, Salomão JCA, Dezen EL, Pinto Filho WC. Fraturas da coluna vertebral em crianças: estudo de 38 casos. Rev Bras Ortop. 1993; 28(3):105-11.

11. Barros Filho TE, Taricco MA, Oliveira RP, Greve JM, Santos LC, Napoli MM. Estudo epidemiológico dos pacientes com traumatismo da coluna vertebral e déficit neurológico, internados no Instituto de Ortopedia e Traumatologia do Hospital das Clínicas da Faculdade de Medicina da USP. Rev Hosp Clin Med Univ São Paulo. 1990; 45(3):123-6.

12. Botelho RV, Abgussen CM, Machado GC, Elias AJ, Silva AA, Bittencourt LR, Fontoura EA. Epidemiologia do trauma raquimedular cervical na zona norte da cidade de São Paulo. Arq Bras Neurocir. 2001; 20(3/4):64-76.

13. Bracken MB, Freeman DH Jr, Hellenbrand K. Incidence of acute traumatic hospitalized spinal cord injury in the United States, 1970-1977. Am J Epidemiol. 1981; 113(6) 615-22.

14. Frankel HL, Hancoock DO, Hyslop G, Melzak J, Michaelis LS, Ungar GH, Vernon JD, Walsh JJ. The value of postural reduction in the initial management of closed injuries of the spine with paraplegia and tetraplegia. I. Paraplegia. 1969; 7(3):179-92.

15. Freitas PE. Traumatismos raquimedulares agudos: estudo epidemiológico de cem casos consecutivos. J Bras Neurocir. 1990; 2(1):1-10.

16. Gehrig R, Michaelis LS. Statistics of acute paraplegia and tetraplegia on a national scale. Paraplegia. 1968; 6(1):93-5.

17. Ingham SJ, Gaspar AP, Vianna PP, Santos FP, Masiero D, Puertas EB. Avaliação epidemiológica dos pacientes com lesão medular atendidos no Lar Escola São Francisco. Coluna/columna. 2004; 3(1):26-9.

18. Masini M, Khan P, Teixeira MM, Plaintier ALMB, Cantanhede WC, Maranhão VL. Trauma raquimedular em crianças - diferenças na etiologia, tratamento e evolução quando comparado com o adulto. Arq Bras Neurocirurg. 1995; 14(2):119-28.

19. McKinley WO, Seel RT, Hardman JT. Nontraumatic spinal cord injury: incidence, epidemiology, and functional outcome. Arch Phys Med Rehabil. 1999; 80(6):619-23.

20. Mello LR, Espíndola G, Silva FM, Bernardes CI. Lesado medular. Estudo prospectivo de 92 casos. Arq Bras Neurocir. 2004; 23(4):151-6.

21. Oliveira PA, Pires JV, Borges Filho JM. Traumatismos da coluna torácica e lombar. Avaliação epidemiológica. Rev Bras Ortop. 1996; 31(9):771-6.

22. Solino JL, Melo MF, Silva DH, Elias N. Traumatismos da coluna vertebral. Avaliação de etiologia, incidência e frequiência. Rev Bras Ortop. 1990; 25(3):185-90.

23. Zaninelli EM, Graells XS, Néri OJ, Dau L. Avaliação epidemiológica das fraturas da coluna torácica e lombar de pacientes atendidos no Pronto-Socorro do Hospital do Trabalhador da UFPR de Curitiba - Paraná. Coluna/Columna. 2005; 4(1):11-5.

24. Kannus P, Niemi S, Palvanen M, Parkkari J. Continuously increasing number and incidence of fall-induced, fractureassociated, spinal cord injuries in elderly persons. Arch Intern Med. 2000; 160(14):2145-9.

25. DeVivo MJ, Rutt RD, Black KJ, Go BK, Stover SL. Trends in spinal cord injury demographics and treatment outcomes between 1973 and 1986. Arch Phys Med Rehabil. 1992; 73(5):424-30. Erratum in: Arch Phys Med Rehabil. 1992;73(12):1146.

26. Carodozo-Gonzales RI, Villa TC, Caliri MH. O processo da assistência ao paciente com lesão medular: gerenciamento de caso como estratégia de organização da alta hospitalar. Medicina Ribeirão Preto. 2001; 34(3/4):325-33.

27. van Asbeck FW, Post MW, Pangalila RF. An epidemiological description of spinal cord injuries in the Netherlands in 1994. Spinal Cord. 2000; 38(7):420-4. 
28. Sposito MM, Laredo Filho J, Braga FM, Novo NF. Paraplegia por lesão medular: estudo epidemiológico em pacientes atendidos para reabilitação. Rev Paul Med. 1986; 104()196-202.

29. Souza MF Jr, Bastos BP, Jallageas DN, Medeiros AA. Perfil epidemiológico de 80 pacientes com traumatismo raquimedular, internados no Hospital do Pronto Socorro Municipal de Belém, PA, no período de janeiro a setembro de 2002. J Bras Neurocirurg. 2002; 13(1):32-8.

30. Kewalramani LS, Kraus JF. Acute Spinal-Cord Lesion from Diving - Epidemiological and Clinical Features. West J Med. 1977; 126(5):353-61.

31. Defino HL, Rodriguez Fuentes AE, Remondi PH, Vallim EC. Tratamento conservador das fraturas da coluna toracolombar. Rev Bras Ortop. 2000; 35(8):301-8.

32. New PW, Sundararajan V. Incidence of non-traumatic spinal cord injury in Victoria, Australia: a population-based study and literature review. Spinal Cord. Epub 2007 Dec 11.
33. Barss P, Djerrari H, Leduc BE, Lepage Y, Dionne CE. Risk factors and prevention for spinal cord injury from diving in swimming pools and natural sites in Quebec, Canada: a 44-year study. Accid Anal Prev. 2008; 40(2):787-97. Epub 2007 Oct 8.

Como citar este artigo:

Campos MF, Ribeiro AT, Listik S, Pereira CA, Sobrinho JA, Rapoport A. Epidemiologia do traumatismo da coluna vertebral no serviço de neurocirurgia do hospital Heliópolis, São Paulo, Brasil. Rev Col Bras Cir. [periódico na Internet] 2008; 35(2). Disponível em URL: http:/ /www.scielo.br/rcbc

Endereço para correspondência:

Departamento de Neurocirurgia do Hospital Heliópolis, Hosphel, São Paulo.

Dr. Marcelo Ferraz de Campos

Rua Cônego Xavier, 276 - $9^{\circ}$ andar.

04231-030 - São Paulo - SP.

E-mail: ferrazcampos@uol.com.br 